\title{
Long-Term Survival, Quality of Life, and Psychosocial Outcomes in Advanced Melanoma Patients Treated with Immune Checkpoint Inhibitors
}

\author{
Anne Rogiers $\left(\mathbb{D},{ }^{1}\right.$ Annelies Boekhout, ${ }^{2}$ Julia K. Schwarze, ${ }^{3}$ Gil Awada, ${ }^{3}$ \\ Christian U. Blank, ${ }^{2}$ and Bart Neyns $\mathbb{1}^{3}$ \\ ${ }^{1}$ Department of Psychiatry, CHU Brugmann, 1020 Brussels, Belgium \\ ${ }^{2}$ Netherlands Cancer Institute, 1066 CX Amsterdam, Netherlands \\ ${ }^{3}$ Department of Medical Oncology, UZ Brussels, 1090 Brussels, Belgium \\ Correspondence should be addressed to Anne Rogiers; anne.rogiers@chu-brugmann.be
}

Received 28 October 2018; Accepted 25 March 2019; Published 10 April 2019

Guest Editor: Subash C. Gupta

Copyright (C) 2019 Anne Rogiers et al. This is an open access article distributed under the Creative Commons Attribution License, which permits unrestricted use, distribution, and reproduction in any medium, provided the original work is properly cited.

Immune checkpoint inhibitors have become a standard of care option for the treatment of patients with advanced melanoma. Since the approval of the first immune checkpoint (CTLA-4) inhibitor ipilimumab in 2011 and programmed death-1 (PD-1) blocking monoclonal antibodies pembrolizumab and nivolumab thereafter, an increasing proportion of patients with unresectable advanced melanoma achieved long-term overall survival. Little is known about the psychosocial wellbeing, neurocognitive function, and quality of life (QOL) of these survivors. Knowledge about the long term side-effects of these novel treatments is scarce as long-term survivorship is a novel issue in the field of immunotherapy. The purpose of this review is to summarize our current knowledge regarding the survival and safety results of pivotal clinical trials in the field of advanced melanoma and to highlight potential long-term consequences that are likely to impact psychosocial wellbeing, neurocognitive functioning, and QOL. The issues raised substantiate the need for clinical investigation of these issues with the aim of optimizing comprehensive health care for advanced melanoma survivors.

\section{Introduction}

Up to 2010, no medical therapy investigated in a randomized clinical trial had shown to significantly improve overall survival (OS) for patients with unresectable advanced melanoma [1]. Less than half of all patients diagnosed with metastatic melanoma (AJCC stage IV) survived for more than 1 year and only $20 \%$ of all patients were alive after 3 years. However, prior to the development of the currently available lifeprolonging medical therapies, a small percentage of patients with advanced melanoma experienced long-term survival for more than 5 years. The characteristics of this small subpopulation have never been fully elucidated. Patients with natural indolent evolution of metastatic disease and cases suspect of "spontaneous immune mediated remission" (often coincident with the development of vitiligo) are likely to have contributed to this historical "tail of the survival curve" for stage IV melanoma. In addition, complete resection of oligometastatic stage IV disease can occasionally provide durable remission in a small proportion of patients, but identifying these patients prospectively on objective clinical or histopathological characteristics has not been achieved and requires further investigation. Finally, durable remissions and long-term survival following conventional cytotoxic chemotherapy (e.g., dacarbazine, temozolomide) have also been reported in exceptional cases, most often after a complete response (CR) had occurred [2].

In the 1980s, it was established that a small percentage of patients with favorable baseline characteristics who were treated with high-dose interleukin-2 (IL-2) could achieve a durable complete remission. In a comprehensive review of the outcome of 270 patients with unresectable melanoma ( 8 clinical trials conducted between 1985 and 1993), receiving IL2 administered at a high dose resulted in a complete response 
(CR) in 6\% and a partial response (PR) in an additional 10\% of patients. A CR seemed a prerequisite for durable progressionfree survival (PFS) as the median response duration in patients obtaining a PR was limited to 5.9 months. These IL-2 treatment regimens were associated with substantial toxicity with grade 5 adverse events (AE) occurring in $2 \%$ of patients. The two baseline predictive factors for response to high-dose IL-2 therapy were the performance status and whether patients had received prior systemic therapy. Combination regimens of IL-2, interferon- $\alpha$ (IFN- $\alpha$ ), and cisplatin-based combination chemotherapies, while showing high overall response rates with some durable remissions, failed to significantly improve survival rates for patients with advanced melanoma and were subsequently abandoned [3].

Since 2010 effective systemic therapies have become available that improved OS of patients with advanced melanoma. Effective new therapies target the T-cell inhibitory immune checkpoint receptors (including the cytotoxic T-lymphocyteassociated antigen 4 (CTLA-4) and Programmed Death 1 (PD-1) receptors on lymphocytes), or the MAPK-signaling pathway in patients with $B R A F^{V 600}$ mutant melanoma, as well as more recently talimogene laherparepvec (T-VEC, the first approved oncolytic virotherapy for cancer offering a survival benefit in patients with stage IV-M1a). Since 2010, all phase III studies conducted with these new agents have reached their primary endpoint, demonstrating improved OS and thereby revolutionizing the treatment options for patients with unresectable advanced melanoma.

\section{Ipilimumab}

The first systemic treatment ever to significantly improve OS for patients with unresectable advanced melanoma was the CTLA-4 blocking monoclonal antibody ( $\mathrm{mAb}$ ) ipilimumab. This drug was approved in 2011 based on the study outcomes of two randomized phase III trials. The first trial, CA184-002, compared ipilimumab (administered at a dose of $3 \mathrm{mg} / \mathrm{kg}$ intravenously [IV] every 3 weeks for a total of four consecutive doses) to a gp100 vaccine or the combination of both in HLA-2 positive patients with pretreated advanced melanoma [4]. For patients, with stable disease after at least 12 weeks of treatment, and who subsequently were diagnosed with progression of disease, reinduction with ipilimumab was allowed. The objective tumor responses according to the Response Criteria in Solid Tumors (RECIST) criteria ranged from $5.7 \%$ to $11.0 \%$ in the ipilimumab treatment arms. The median OS was improved to 10.0 months for the ipilimumab monotherapy-arm as compared to 6.4 months for the peptide vaccine-alone arm (HR 0.68; $\mathrm{p}<0.001$ ) (Figure 1). Combination of ipilimumab with the gp-100 vaccine provided no benefit over ipilimumab alone (Table 1).

In a second pivotal phase III study (CA184-024), ipilimumab (administered at a dose of $10 \mathrm{mg} / \mathrm{kg}$ every 3 weeks for a total of four consecutive doses and subsequently once every 12 weeks) was combined with dacarbazine chemotherapy $\left(850 \mathrm{mg} / \mathrm{m}^{2}\right)$ and compared with dacarbazine plus placebo. Median OS was improved for ipilimumab plus dacarbazine (11.2 months) as compared to dacarbazine alone (9.1 months; HR 0.72; $\mathrm{p}$ 0.001) (Table 1, Figure 1). The co-administration

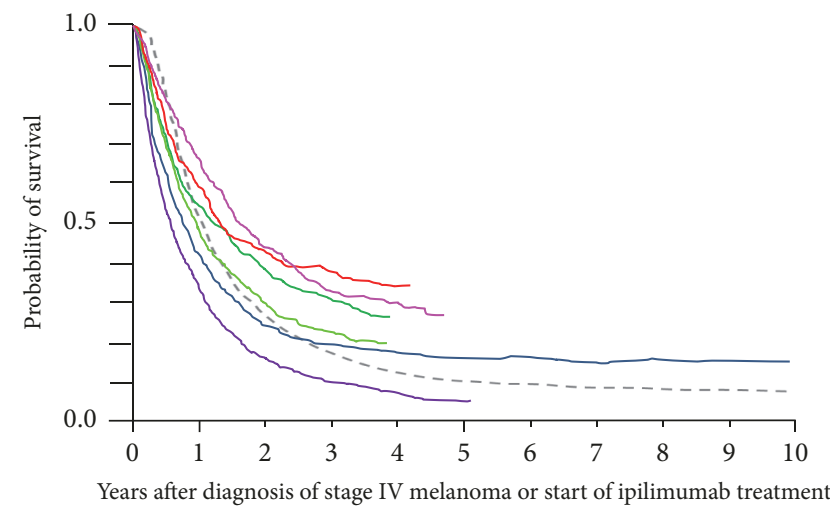

$$
\begin{aligned}
& \text { - (6) CM-067, ipilimumab } 3 \mathrm{mg} / \mathrm{kg}(\mathrm{n}, 315) \\
& - \text { (5) KN-006, ipilimumab } 3 \mathrm{mg} / \mathrm{kg}(\mathrm{n}, 278) \\
& - \text { (4) CA184-367, ipilimumab } 10 \mathrm{mg} / \mathrm{kg}(\mathrm{n}, 365) \\
& - \text { (4) CA184-367, ipilimumab } 3 \mathrm{mg} / \mathrm{kg}(\mathrm{n}, 362) \\
& - \text { (3) EURO-VOYAGE, ipilimumab } 3 \mathrm{mg} / \mathrm{kg}(1,043 \text { patients) } \\
& \text { - (2) Pooled analysis, ipilimumab } 3 \mathrm{mg} / \mathrm{kg} \text { (4,846 patients) } \\
& - \text { - (1) Historical OS from diagnosis of stage IV melanoma }
\end{aligned}
$$

FigURE 1: Overlay of Kaplan-Meier curves indicating the probability for overall survival (OS) for patients treated with ipilimumab as first line of immunotherapy, representing (1) the historical probability for OS for patients diagnosed with stage IV melanoma prior to the availability of life-prolonging medical treatment options (dashed black line) [1]; (2) a pooled OS analysis including individual patient survival data from 1,861 patients with metastatic melanoma from 12 clinical investigations of ipilimumab and 2,985 patients with metastatic melanoma from a US ipilimumab EAP (total $n=4,846)$ (dark blue line) [9]; (3) interim results from EURO-VOYAGE, a multicenter, observational, retrospective study of 1043 patients with advanced melanoma who participated in the EU ipilimumab EAP (purple line) [69]; (4) intention-to-treat population $(365+362$ patients) of the CA184-367 study comparing ipilimumab at $10 \mathrm{mg} / \mathrm{kg}$ (dark green line) to $3 \mathrm{mg} / \mathrm{kg}$ dosing level (light green line) [8]; (5) intention-to-treat population (278 patients) on the ipilimumab arm from the Keynote-006 trial (red line) [13]; (6) intention-totreat population (315 patients) on the ipilimumab arm from the Checkmate-067 trial (pink line) [72].

of ipilimumab with dacarbazine significantly increased the incidence of grade 3 or 4 toxicity hepatic toxicity (grade 3 or 4 AEs occurred in $56.3 \%$ of patients treated with ipilimumab plus dacarbazine, as compared with $27.5 \%$ treated with dacarbazine and placebo) and hepatotoxicity in particular (grade 3 or 4 elevations in liver-function values noted in 17.4 to $20.7 \%$ of the patients) [5].

Additional evidence for the long-term beneficial survival effect from ipilimumab came from a large randomized phase II trial in pretreated patients comparing the $0.3,3$, and $10 \mathrm{mg} / \mathrm{kg}$ dose levels, indicating a dose-dependent outcome in terms of objective tumor response rate and survival, but also a dose-dependent increase in toxicity [65]. In 2011, ipilimumab received approval by the competent authorities in Europe, the US, and Australia for the treatment of advanced melanoma at a dose of $3 \mathrm{mg} / \mathrm{kg}$ administered every 3 weeks for a total of four consecutive doses. The label did not include a reference to the possibility of retreating patients who responded to the initial four doses. Although only a small proportion of 


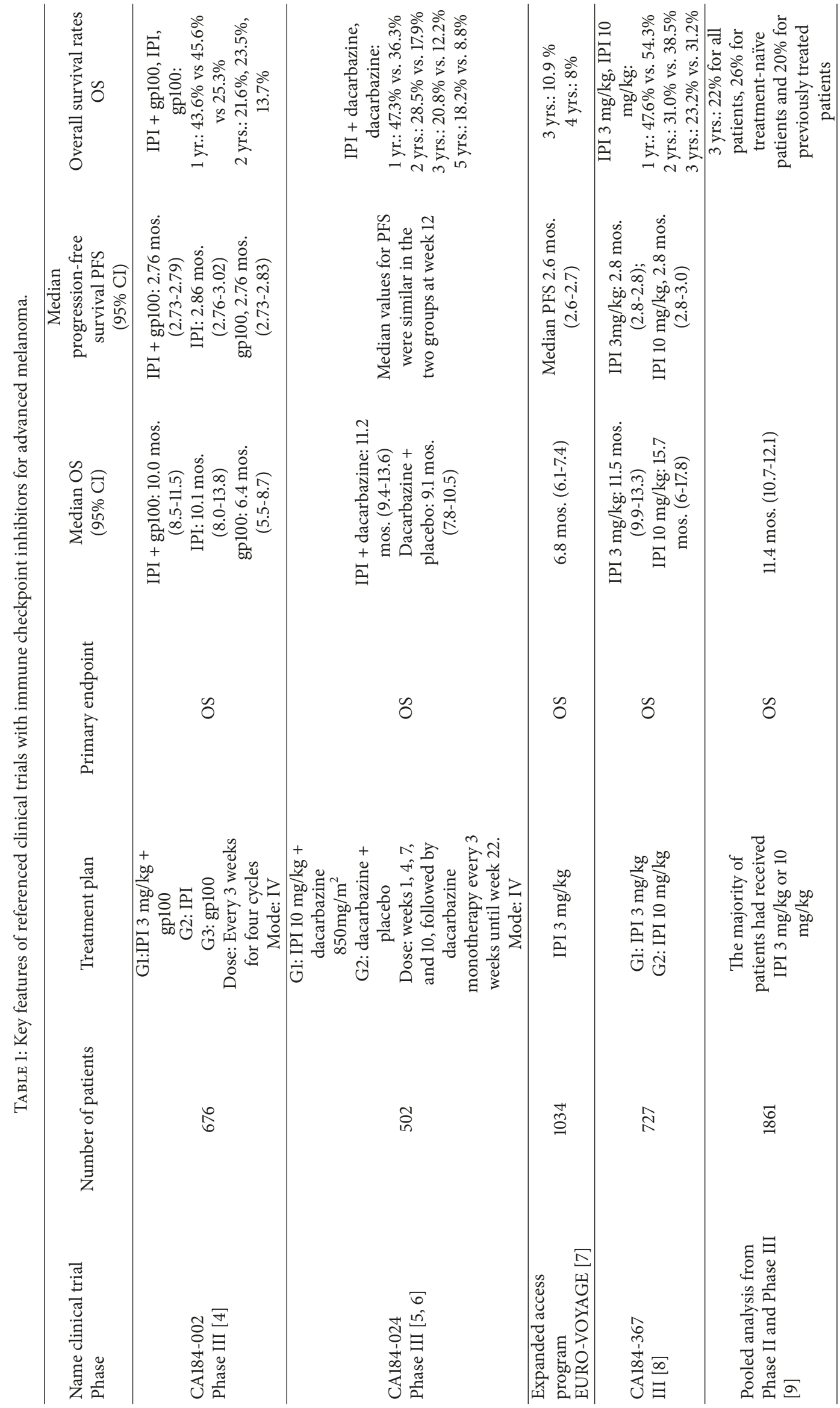




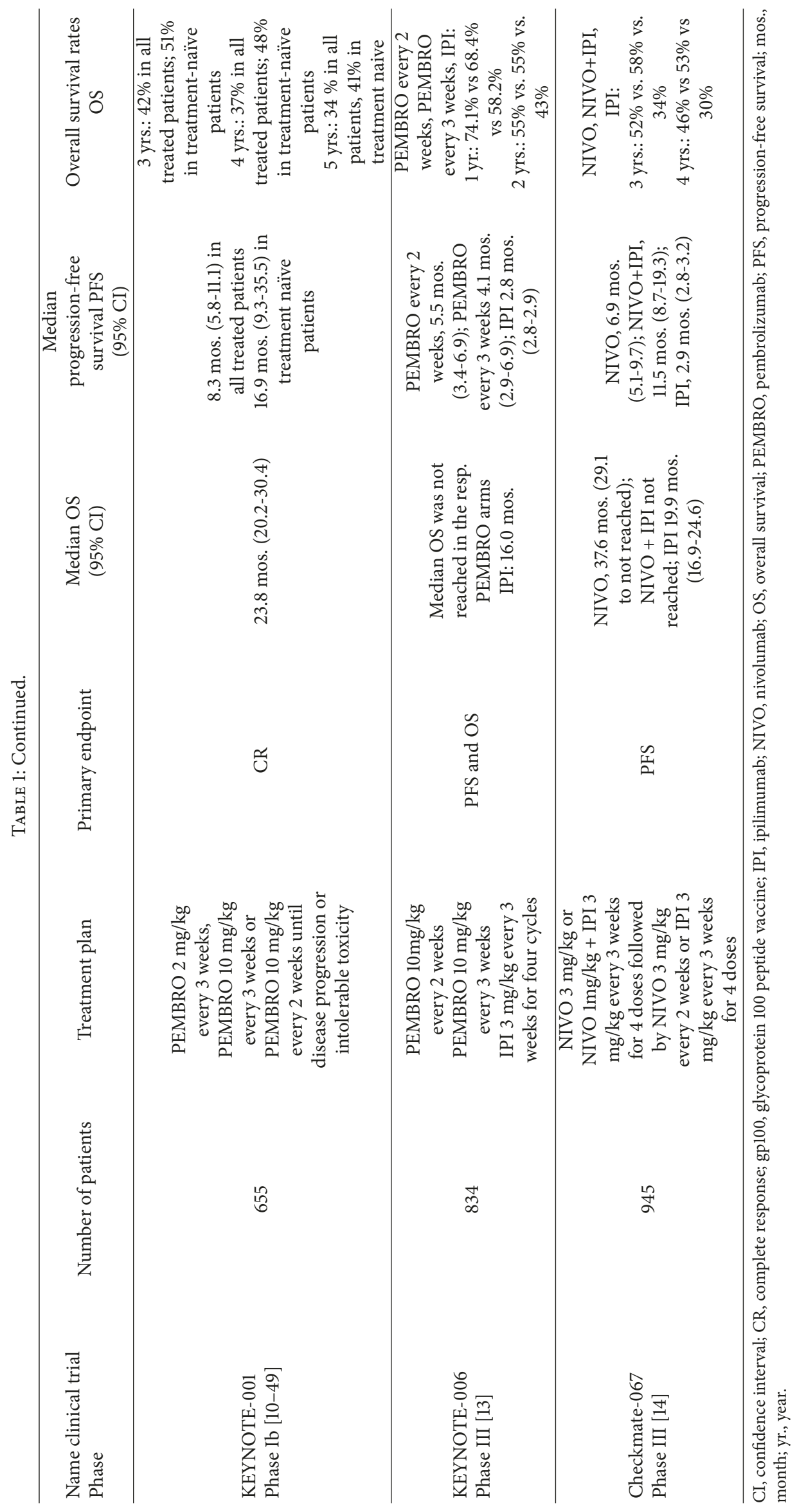


the CA184-002 study population was retreated at the time of first progression following an initial favorable response to ipilimumab, this may have contributed to the long-term ( $\geq 3$ year) survival results on ipilimumab monotherapy [66] (Figure 1).

Novel features of ipilimumab therapy included an increased potential for long-term survival benefit in a small proportion of patients, the occurrence of new adverse events (AEs), the so-called "immune-related AEs" (irAE), and the atypical kinetics of treatment response $[67,68]$. A consistent finding across these clinical trials investigating ipilimumab was the absence of a measurable impact on OS in the first 3 to 4 months of treatment. With longer followup a moderate improvement of the median OS outcome became apparent, and the long-term probability for survival after 3 years or longer (the so-called "tails of the survival curves") was not reconverting, indicative of the fact that $10-15 \%$ of the ipilimumab treated population derived a highly durable survival benefit as compared to the control population. Mature survival data were reported in an updated report of survival rate of the CA184-014 trial and a pooled analysis of 1861 patients from 10 prospective and two retrospective studies: $5 \mathrm{y}$-OS rate was $18.2 \%$ (95\% CI, $13.6 \%$ to $23.4 \%$ ) for patients treated with ipilimumab plus dacarbazine versus 8.8\% (95\% CI, 5.7\% to $12.8 \%$ ) for patients treated with placebo plus dacarbazine $(\mathrm{P}=.002)$ (Table 1). An "inflexion-point" on the curve followed by a plateau in the survival curve began at approximately 3 years [6] (Figure 1).

These findings were confirmed in a pooled analysis including 1861 patients from ten prospective and two retrospective studies, including a majority of patients receiving ipilimumab according to the $3 \mathrm{mg} / \mathrm{kg}(\mathrm{n}=965)$ or $10 \mathrm{mg} / \mathrm{kg}$ $(\mathrm{n}=706)$ dose levels (Table 1). Twenty-two percent of the patients were alive at 3 years, and a plateau on the survival curve became apparent 3 years after the start of treatment [9]. A second analysis of OS data with a total of $n=4,846$ patients (including an additional 2,985 patients from an expanded access program) further confirmed a survival plateau at $21 \%$ from 3 years on (Figure 1).

Following approval of ipilimumab in 2011, a phase III trial (CA184-367), was conducted to address the unresolved question regarding the optimal dosing of ipilimumab (3 vs. $10 \mathrm{mg} / \mathrm{kg}$ ); 727 patients without prior exposure to BRAF or PD-1 inhibitors were randomly assigned (1:1) to ipilimumab with either dose level [8]. The median number of doses of ipilimumab administered was four in each arm, with retreatment being pursued in a minority of patients $(6 \%$ and $9 \%$ of patients in the 10 and $3 \mathrm{mg} / \mathrm{kg}$ arms, respectively). The median OS was superior for patients treated on the $10 \mathrm{mg} / \mathrm{kg}$ arm (15.7 versus 11.5 months; HR 0.84; $\mathrm{p}=0.04$ ) (Table 1). No difference in the probability for survival was evident during the first 6 months of followup. Thereafter the curves separated and a distinct 2- and 3-year survival rate was observed between both dose levels of ipilimumab (Figure 1). Treatment-related AEs in the $10 \mathrm{mg} / \mathrm{kg}$ arm were more frequent as compared to the $3 \mathrm{mg} / \mathrm{kg}$ arm $(79 \%$ allgrade and $34 \%$ grade 3 to $5 \mathrm{AEs}$, as compared with $54 \%$ and $14 \%)$.
More recently, the effectiveness of ipilimumab was examined in a systematic retrospective analysis of 1034 patients with advanced melanoma who were included in a European Expanded Access Program (EURO-VOYAGE). A median OS of 6.8 months was found and the 3- and 4-years OS rates were, respectively, 10.9 and $8 \%$ and thus were apparently lower than what had been reported before (Table 1) [69]. These results indicate that the level of the "tail of the survival curve" remains dependent on the baseline characteristics of the investigated population, with an important role for baseline covariables as determinants for durable survival for patients treated with ipilimumab (Figure 1) [7, 70, 71].

\section{Anti-PD-1 Therapies}

Since 2015, ipilimumab has been replaced as the preferred first choice immunotherapy for advanced melanoma by PD-1 blocking mAb. Pembrolizumab and nivolumab were approved in the EU, US, and Australia as first-line immunotherapy for advanced melanoma based on phase III trials demonstrating a significant improvement of both PFS and OS as compared to ipilimumab [74,75]. Notwithstanding the relative short followup of up to 3-4 years for these study populations, superior survival rates have been reported at every land-mark analysis [72, 76]. Moreover, followup of patient populations treated on phase I trials with nivolumab and pembrolizumab have also demonstrated the potential for durable survival gains after up to 5 years of followup $[10,11]$. Anti-PD1 therapies are associated with a lower incidence of immune-related AEs as compared to ipilimumab [12].

3.1. Pembrolizumab. In the KEYNOTE-006 phase III trial, patients with unresectable stage III or IV melanoma had been randomly assigned (1:1:1) to one of two dose regimens of pembrolizumab $(10 \mathrm{mg} / \mathrm{kg}$ every 2 or 3 weeks $)$ or one regimen of ipilimumab ( $3 \mathrm{mg} / \mathrm{kg}$ every 3 weeks for a total of 4 consecutive doses) [13] (Table 1). Pembrolizumab treatment was continued for a maximum duration of 2 years. After a median followup of 22.9 months, median OS was not reached in either pembrolizumab group and was 16.0 months with ipilimumab (hazard ratio [HR] $0.68,95 \%$ CI 0.53-0.87 for pembrolizumab every 2 weeks vs. ipilimumab $p=0.0009$; and $0.68,0.53-0.86$ for pembrolizumab every 3 weeks vs. ipilimumab; $\mathrm{p}=0.0008$ ) with a 24 -month OS rate of $55 \%$ for pembrolizumab treated patients and $43 \%$ in the ipilimumab group. The 33 -month PFS-rate was 31 vs. $14 \%$ and OS-rate 50 vs. $39 \%$ for the pooled pembrolizumab arms vs. the ipilimumab group. After a median followup of 45.9 months (range: $0.3-50.0$ ) the 4 -year OS rates were $42 \%$ in the pooled pembrolizumab groups and $34 \%$ in the ipilimumab group (Figure 2). One hundred and three patients (19\%) received the maximum duration of 2 years of pembrolizumab treatment and only $14 \%$ of the patients experienced progressive disease (median followup of 20.3 months) [13].

In an open-label phase $1 \mathrm{~b}$ clinical trial (KEYNOTE-001) patients received pembrolizumab $2 \mathrm{mg} / \mathrm{kg}$ or $10 \mathrm{mg} / \mathrm{kg}$ every 3 weeks or $10 \mathrm{mg} / \mathrm{kg}$ every 2 weeks until disease progression or intolerable toxicity. The median OS was 23.8 months in all 655 patients, with 3-year and 4-year survival estimates of $42 \%$ and 


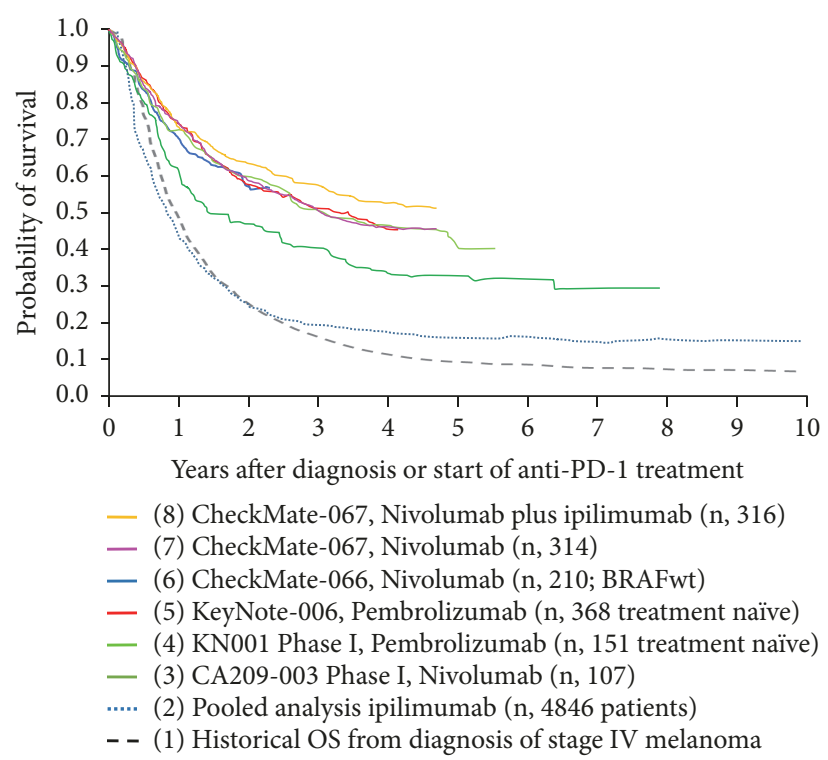

FIGURE 2: Overlay of Kaplan-Meier curves indicating the probability for OS (OS) for advanced melanoma patients treated with anti-PD1 as first-line immunotherapy, representing (1) the historical probability for OS for patients diagnosed with stage IV melanoma prior to the availability of life-prolonging medical treatment options (dashed black line) [1]; (2) a pooled OS analysis including individual patient survival data from 1,861 patients with metastatic melanoma from 12 clinical investigations of ipilimumab and 2,985 patients with metastatic melanoma from a US ipilimumab EAP (total $n=4,846$ ) (blue line) [9]; (3) CA209-003 phase I clinical trial with nivolumab for pretreated advanced melanoma patients (dark green line) [11]; (4) treatment naïve patients (n: 151) treated in the Keynote-001 clinical trial with pembrolizumab (light green line) [10]; (5) treatment naïve patients ( $n, 368$ ) from the Keynote-006 trial (red line) [13]; (6) nivolumab treated patients with BRAF V600 wild-type melanoma (n, 210) from the Checkmate-066 trial (blue line) [73]; (7) nivolumab monotherapy treated patients $(\mathrm{n}, 314)$ from the CheckMate-067 trial (pink line) [72]; (8) nivolumab plus ipilimumab treated patients (n, 316) from the CheckMate-067 trial (orange line) [72].

37\% (Table 1). In the 152 treatment-naive patients, the 3-year and 4 -year survival estimates were $51 \%$ and $48 \%$, respectively (Table 1). Recently the updated 5 years overall survival results have been published and an OS of 34\% in all patients and an OS of $41 \%$ in treatment-naïve patients were found (Figure 2) $[10,76,77]$.

3.2. Nivolumab. Similar survival outcome has been observed in another double-blind, phase 3 study, investigating nivolumab alone or nivolumab plus ipilimumab versus ipilimumab alone as first line therapy in 945 previously untreated patients with unresectable stage III or IV melanoma (Checkmate-067) [14]. Both nivolumab containing treatment arms significantly improved both PFS and OS as compared to ipilimumab and a superior PFS was obtained in the combination arm of nivolumab and ipilimumab (Table 1). However, treatmentrelated AEs of grade 3 or 4 occurred more frequently with upfront combination of nivolumab and ipilimumab $(22.4 \%$ of the patients in the nivolumab monotherapy arm, 59.1\% of those in the combination arm, and $27.7 \%$ of those in the ipilimumab monotherapy arm). After a minimum followup of 48 months, the median OS had not been reached in the combination group and was 36.9 months in the nivolumab monotherapy group, as compared with 19.9 months in the ipilimumab monotherapy group (hazard ratio for death with nivolumab plus ipilimumab vs. ipilimumab, $0.54[\mathrm{P}<0.001]$; hazard ratio for death with nivolumab vs. ipilimumab, 0.65 $[\mathrm{P}<0.001])$. The OS rate at 4 years was $53 \%$ in the nivolumabplus-ipilimumab and $46 \%$ in the nivolumab monotherapy arm, as compared with $30 \%$ in the ipilimumab monotherapy arm (Figure 2). The two groups including nivolumab had significantly longer survival compared to the ipilimumab group. In a descriptive analysis, the hazard ratio for death with nivolumab plus ipilimumab versus nivolumab monotherapy was not statistically significant (hazard ratio for death was 0.84 with a $95 \%$ CI, 0.67 to 1.05 ).

Comparable to patients treated with pembrolizumab, the hazard ratio for progression of disease decreased with time and the rate of PFS at 4 years was 37\% in the nivolumab-plusipilimumab group and $31 \%$ in the nivolumab monotherapy arm, as compared with $9 \%$ in the ipilimumab monotherapy arm. In a descriptive analysis, the hazard ratio for progression or death was 0.79 (95\% CI, 0.65 to 0.97 ) with nivolumab plus ipilimumab versus nivolumab indicating the potential for a stable survival plateau above $30 \%$ in both nivolumab treatment arms (Figure 2).

Long-term prediction of OS-rates in patients with advanced melanoma treated with anti-PD-1 mAb is currently only available for pretreated patient populations who participated in phase I clinical trial programs. The available data nevertheless are indicative that the OS probability curve is likely to reach a plateau. Thirty-four percent of patients treated with nivolumab in a phase I trial (CA209-003) were alive 5 years after initiating study treatment [49].

3.3. Real-World Outcome Data on Anti-PD-1 Therapy. In a poster presented at the SMR 2017 annual meeting real-world outcome data were reported on 189 advanced melanoma patients discontinuing anti-PD-1 treatment (pembrolizumab or nivolumab) in the absence of PD or treatment limiting toxicity [15]. Data were collected at 14 hospitals across Europe and Australia. Short-term outcome of patients that stopped therapy in absence of progression of disease or treatment limiting toxicity was encouraging, with a low-risk for PD (4\% after a median FU of 35 weeks). Reintroduction of a PD1-inhibitor in patients who progressed after discontinuation ( $n=9$ patients) indicated the potential for renewed antitumor activity. Additional reports, in line with these results on realworld outcome data, were recently reported by additional groups [16-19].

\section{Long-Term Immune Related Adverse Events}

The side effects of immune checkpoint blockade are often referred to as immune-related adverse events (irAE). The most common irAE occur in skin, liver, and gastrointestinal, pulmonary, and endocrine organs but autoimmune diabetes 
and cardiovascular, renal, and musculoskeletal side effects are also reported $[20,21]$. Most cutaneous, gastrointestinal, and hepatic AEs occurred within two months, whereas endocrine, pulmonary, and renal side effects appeared after 9 weeks [68]. Early diagnosis and treatment are believed to be important in mitigating the severity of irAEs [22]. Most of these irAE are reversible after treatment interruption and/or steroid therapy; however, the endocrine irAE (most commonly hypophysitis and thyroiditis) may necessitate life-long hormonal substitution $[23,24]$. One study on 15 patients diagnosed with autoimmune hypophysitis induced by ipilimumab treatment reported that all patients had at least one hormonal defect at diagnosis [25]. In all patients clinical symptoms improved in the first month after starting glucocorticoid therapy. At the end of followup (median 33.6 months, range 7-53.5), 13 (86.6\%) required long-term hormonal replacement with corticotropic deficiency persisting in all patients suffering from hypocorticism. No prospective study results are currently available on the long-term ( $>3 \mathrm{yrs})$ consequences of irAEs.

The high incidence of irAE observed with the combination of nivolumab $(1 \mathrm{mg} / \mathrm{kg})$ and ipilimumab $(3 \mathrm{mg} / \mathrm{kg})$ has prompted the investigation of nivolumab or pembrolizumab combined with ipilimumab at a lower dose level of $1 \mathrm{mg} / \mathrm{kg}$ every 3 weeks $[26,27]$. The CheckMate 511 study demonstrated a significantly lower incidence of treatment-related grade 3-5 AEs. However, longer followup is needed to address the long-term OS outcome as for ipilimumab in monotherapy, long-term OS is dose dependent.

\section{Health Related Quality of Life in Melanoma Survivors}

To date patient reported global health related quality of life (HRQOL) is measured using several valid instruments to assess different dimensions of HRQOL, such as psychological, social, physical, and spiritual aspects. HRQOL instruments can be generic, cancer specific, or cancer disease specific and measure only one or several dimensions. Commonly used scales to assess the global HRQOL in cancer patients include the European Organization for Research and Treatment of Cancer Quality of life Questionnaire (EORTCQLQ-C30), the Impact of Cancer questionnaire (IOC), and the Generic Functional Assessment of cancer therapy (FACTG) for which an additional melanoma scale was validated, the FACT melanoma (FACT-M) [28-31]. In the field of survivorship the EORTC-QOL survivorship questionnaire is currently in validation process [32].

Results from three randomized controlled trials (MDX010-20, KEYNOTE-002, and CheckMate 067) suggest that ipilimumab, nivolumab, and pembrolizumab, as a monotherapy, and the combination therapy of nivolumab plus ipilimumab or ipilimumab plus gp100 vaccine are well tolerated and either improve or maintain HRQOL as assessed with the EORTC QLQ-C30 scale, during the treatment induction phase [33-35]. However, there might be an underestimation of the influence of these treatments on the HRQOL because of low patient numbers in the later weeks of all studies due to disease progression, death, and AEs. In the KEYNOTE-006 it has been observed that the HRQOL assessed with the EORTC QLQ-C30 scale, in patients treated with pembrolizumab, was better maintained as compared to ipilimumab in patients with ipilimumab naïve advanced melanoma (Table 2) [34].

In a systematic review of 7 studies (4246 patients; 6 cross-sectional [36-44], and 1 prospective study [45]), it was found that determinants of lower HRQOL (either psychological, physical, or global) were marital status, age, sex, poor social support, melanoma severity at diagnosis, and comorbidities (Table 3) [46]. Dieng et al. found that HRQOL, measured with the Functional Assessment of Cancer Therapy (FACT-M), was correlated with fear of recurrence of disease in patients with metastatic melanoma (Table 3) [47]. A more comprehensive understanding of HRQOL can improve patient centered care in melanoma patients. In addition HRQOL assessment can be used as outcome measure for cancer research and help socioeconomic decision making. Therefore international consensus on how to assess HRQOL is mandatory, as well as the development and validation of melanoma specific assessment tools $[46,48]$. In Table 4 an overview is given of the characteristics of the questionnaires used in the referenced trials.

\section{Psychosocial Outcomes in Melanoma Survivors}

With increasing numbers of advanced melanoma patients becoming long-term cancer survivors, even after discontinuing therapy, the issue of melanoma survivorship care becomes of relevance to more patients than ever before. Cancer survivorship has been extensively studied in other cancer indications [49]. In these studies, cancer survivors have been reported to suffer from mental and physical symptoms, fatigue, and neurocognitive dysfunction persisting after physical recovery from their disease. These mental and neurocognitive symptoms are associated with important psychosocial consequences such as delayed return to work, impaired family relationships, and reduced quality of life (QOL) [77, 78].

Only a few studies are focusing on psychosocial outcome in melanoma survivors, with all of them showing diminished wellbeing, high levels of distress, and fear for recurrence (Table 3) [36, 43]. Nevertheless, results of these studies are limited as they are all survey-based and mainly include patients with early stage melanoma and in a lesser extent nonmetastatic disease treated with adjuvant therapy. The following risk factors have been described to be related to higher distress in early stage melanoma patients: female gender, younger age, negative appraisal, and negative coping strategies (Table 3) [63]. Higher distress and fear of recurrence might be related to the necessity of continued self-examination, dermatological controls, and reduced sun exposure [37, 79]. Moreover, higher anxiety levels and fear for recurrence are associated with avoidance behavior in relation to dermatological controls [80]. The traumatic course of metastatic melanoma may also contribute to more difficult coping mechanism as compared to other cancer indications [46]. In accordance with these findings, Dieng explored 


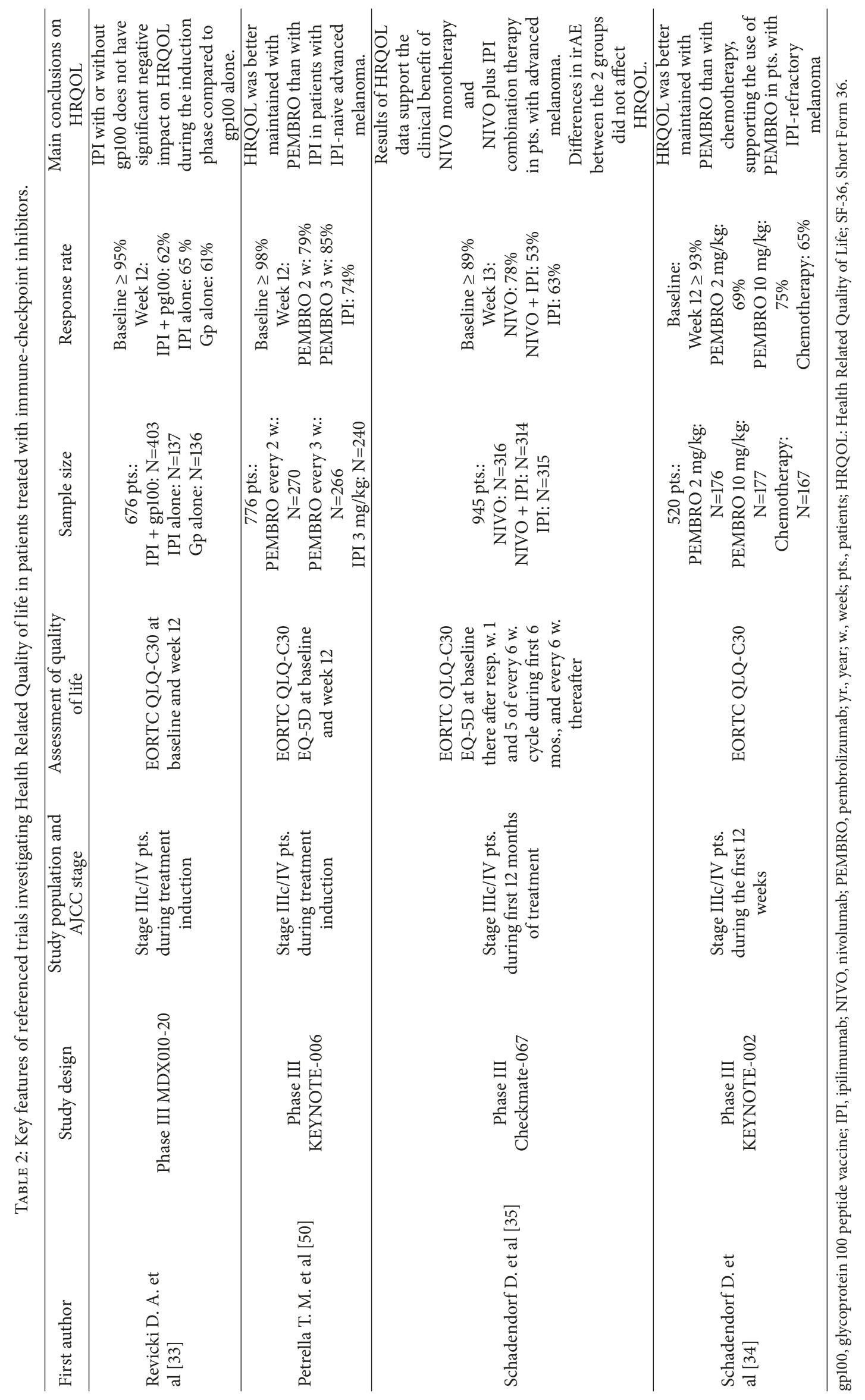




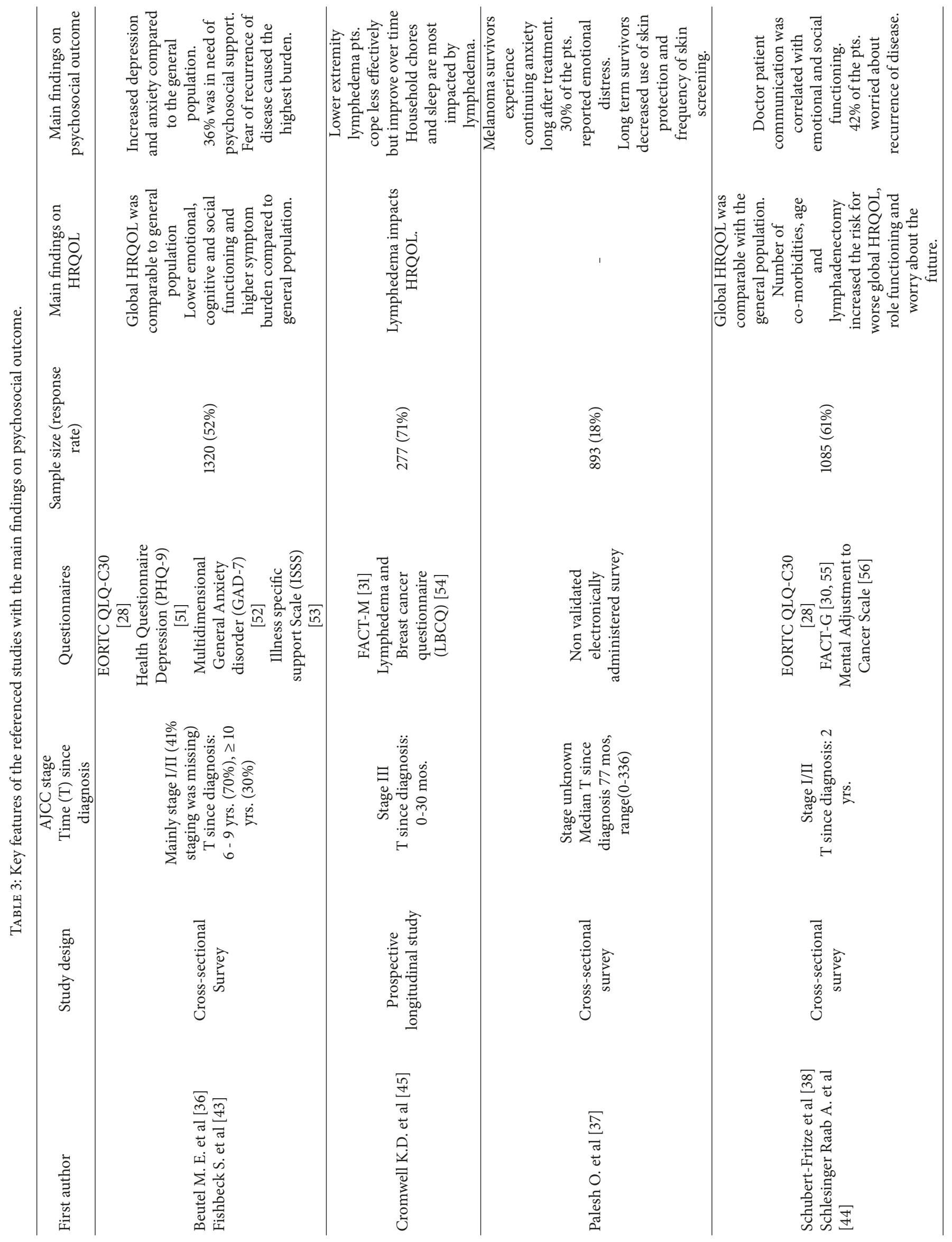




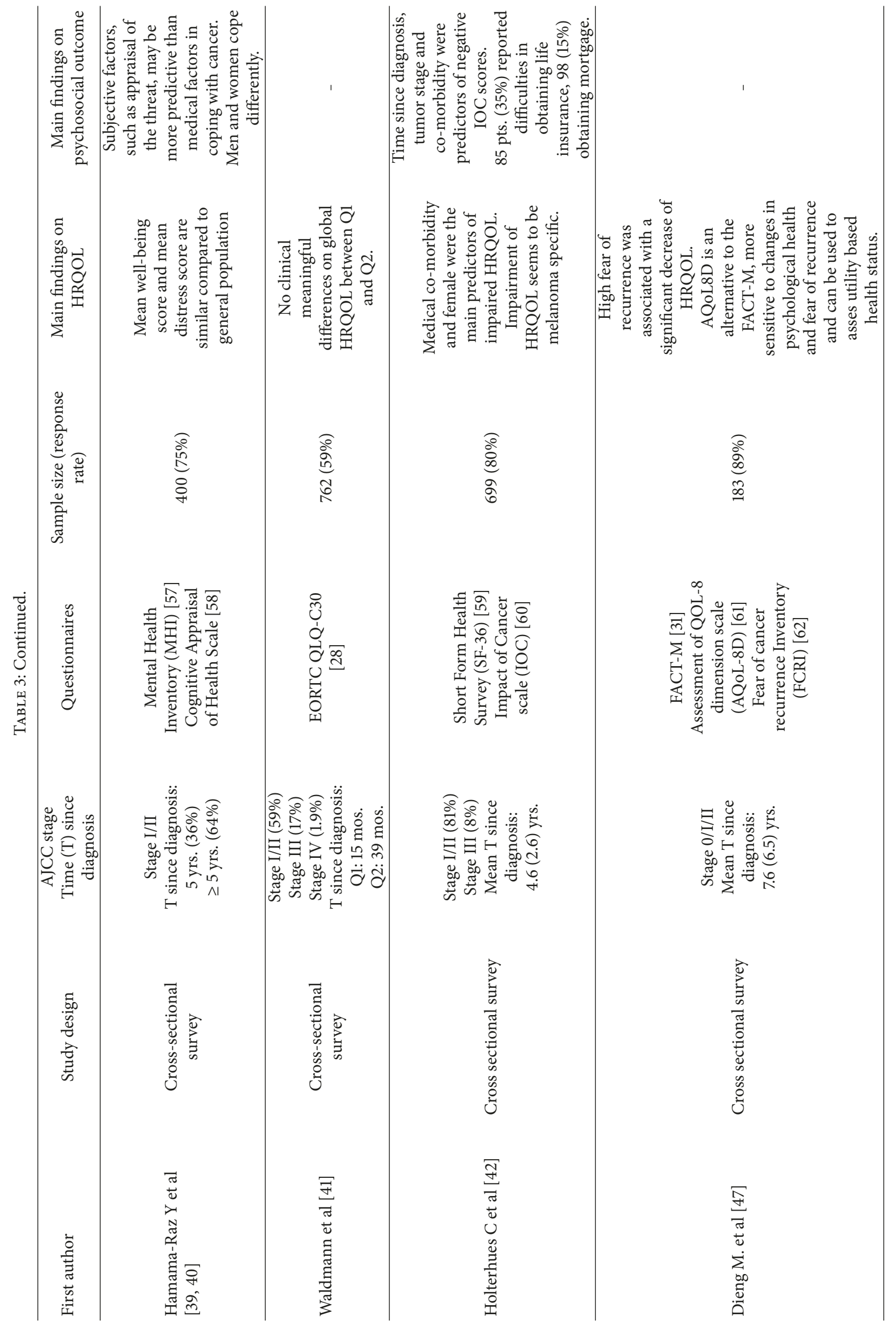




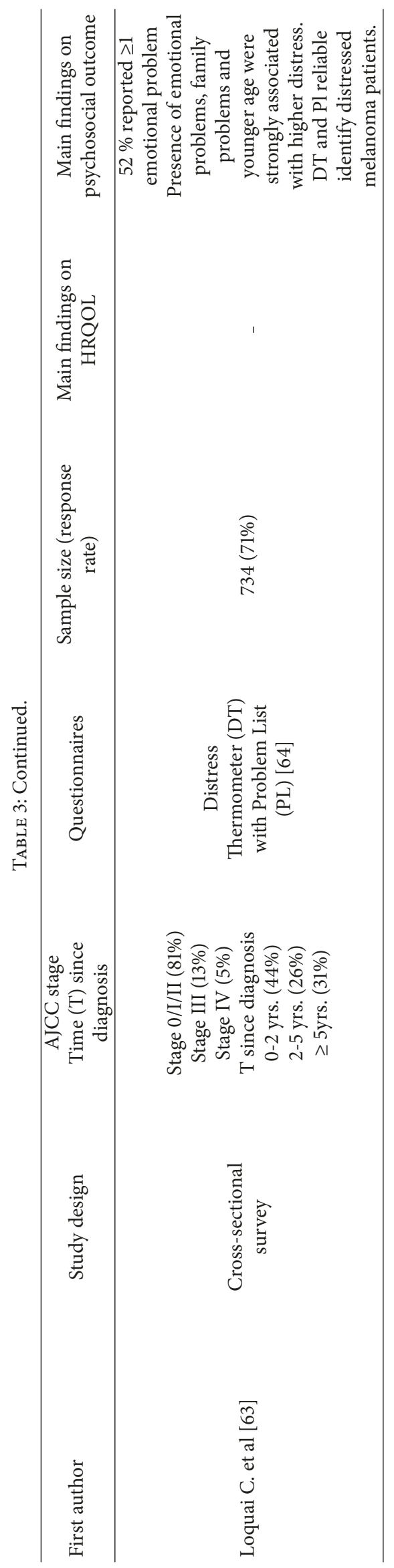




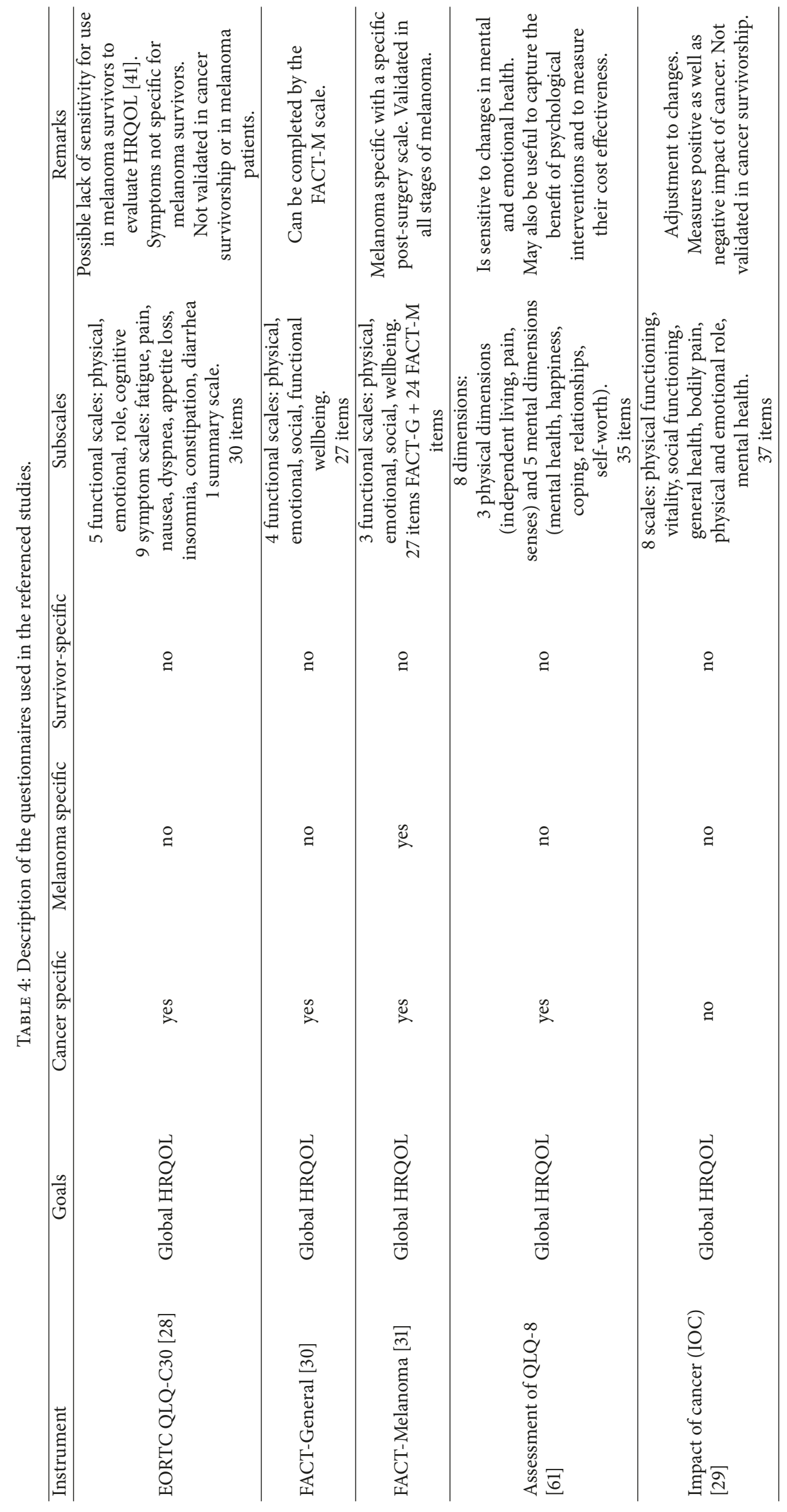




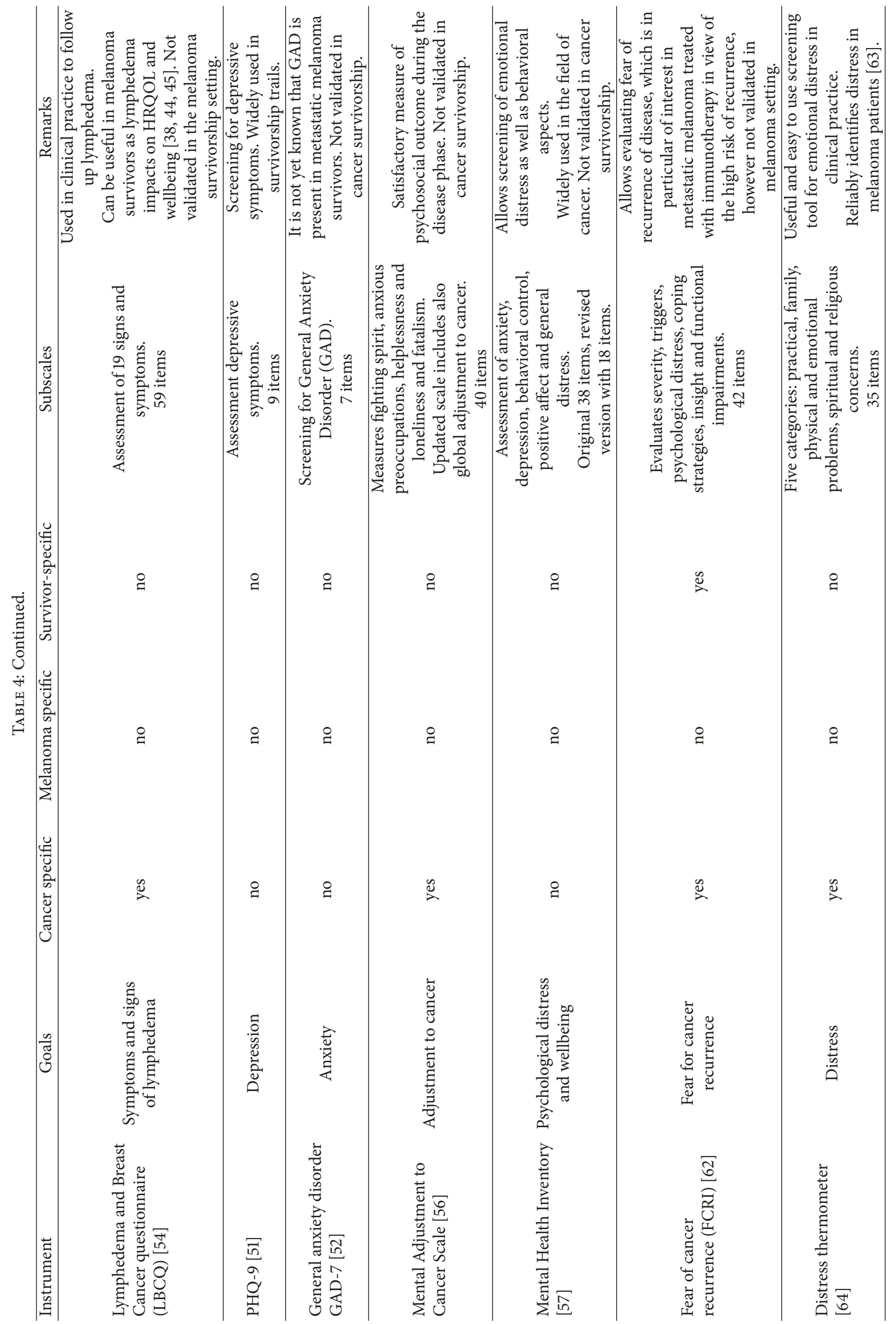


the usefulness of psychoeducational intervention in patients diagnosed with stages 1-2 melanoma and found a substantial benefit compared to the patients who received standard of care [81].

Currently no data are available on the potential longterm emotional, physical and cognitive side effects of immune checkpoint inhibitors in patients with metastatic melanoma.

Moreover, an important subgroup of patients with brain metastasis is becoming survivors, which makes it imperative to study potential effects on neurocognitive functioning, especially because survivors who have previously been irradiated for brain metastases are at increased risk for focal postradiation necrosis of the brain [82]. Efforts to further comprehensively address these psychosocial, neurocognitive, and HRQOL issues are ongoing at present at our department. Preliminary observations indicate that a substantial fraction of these patients experience diminished HRQOL, persisting fatigue, severe emotional disturbances, and neurocognitive complaints $[83,84]$. A multicentric study addressing HRQOL in long-term survivors following treatment with ipilimumab is currently ongoing in The Netherlands and Belgium.

In conclusion prospective investigation of the potential psychosocial, neurocognitive, and HRQOL issues is needed, in order to identify the care needs of advanced melanoma survivors. Optimizing patients' subjective wellbeing could potentially reduce the emotional, physical, and socioeconomic consequences of this devastating disease.

\section{Conflicts of Interest}

Anne Rogiers reports personal fees from BMS and MSD, outside the submitted work. Christian U. Blank reports personal fees from MSD, BMS, Roche, GSK, Novartis, Pfizer, GenMab, Pierre Fabre, and Lilly and grants from BMS, NanoString, and Novartis, outside the submitted work. Bart Neyns reports personal fees from BMS, MSD, Amgen, Pfizer, Roche, and Merk/Serono, outside the submitted work. Annelies Boekhout, Gil Awada, and Julia Schwarze declare that they have no conflicts of interest.

\section{References}

[1] H. Tsao, M. B. Atkins, and A. J. Sober, "Management of cutaneous melanoma," The New England Journal of Medicine, vol. 351, no. 10, pp. 998-1042, 2004.

[2] C. Kim, C. W. Lee, L. Kovacic, A. Shah, R. Klasa, and K. J. Savage, "Long-term survival in patients with metastatic melanoma treated with DTIC or temozolomide," The Oncologist, vol. 15, no. 7, pp. 765-771, 2010.

[3] M. B. Atkins, J. Hsu, S. Lee et al., "Phase III trial comparing concurrent biochemotherapy with cisplatin, vinblastine, dacarbazine, interleukin-2, and interferon alfa-2b with cisplatin, vinblastine, and dacarbazine alone in patients with metastatic malignant melanoma (E3695): a trial coordinated by the Eastern Cooperative Oncology Group," Journal of Clinical Oncology, vol. 26, no. 35, pp. 5748-5754, 2008.

[4] F. S. Hodi, S. J. O’Day, D. F. McDermott et al., "Improved survival with ipilimumab in patients with metastatic melanoma," The New England Journal of Medicine, vol. 363, no. 13, pp. 711723, 2010.
[5] C. Robert, L. Thomas, I. Bondarenko et al., "Ipilimumab plus dacarbazine for previously untreated metastatic melanoma," The New England Journal of Medicine, vol. 364, no. 26, pp. 25172526, 2011.

[6] M. Maio, J.-J. Grob, S. Aamdal et al., "Five-year survival rates for treatment-naive patients with advanced melanoma who received ipilimumab plus dacarbazine in a phase III trial," Journal of Clinical Oncology, vol. 33, no. 10, pp. 1191-1196, 2015.

[7] S. Wilgenhof, S. D. Four, F. Vandenbroucke et al., "Single-center experience with ipilimumab in an expanded access program for patients with pretreated advanced melanoma," Journal of Immunotherapy, vol. 36, no. 3, pp. 215-222, 2013.

[8] P. A. Ascierto, M. Del Vecchio, C. Robert et al., "Ipilimumab 10 $\mathrm{mg} / \mathrm{kg}$ versus ipilimumab $3 \mathrm{mg} / \mathrm{kg}$ in patients with unresectable or metastatic melanoma: a randomised, double-blind, multicentre, phase 3 trial," The Lancet Oncology, vol. 18, no. 5, pp. 611622, 2017.

[9] D. Schadendorf, F. S. Hodi, C. Robert et al., "Pooled analysis of long-term survival data from phase II and phase III trials of ipilimumab in unresectable or metastatic melanoma," Journal of Clinical Oncology, vol. 33, no. 17, pp. 1889-1894, 2015.

[10] O. Hamid, C. Robert, A. Daud et al., "5-year survival outcomes in patients (pts) with advanced melanoma treated with pembrolizumab (pembro) in KEYNOTE-001," Journal of Clinical Oncology, vol. 36, no. suppl 15, p. 9516, 2018.

[11] F. S. Hodi, H. Kluger, M. Sznol et al., "Abstract CT001: Durable, long-term survival in previously treated patients with advanced melanoma (MEL) who received nivolumab (NIVO) monotherapy in a phase I trial:", Cancer Research, vol. 76, supplement 14, p. CT001, 2016.

[12] J. S. Weber, F. S. Hodi, J. D. Wolchok et al., "Safety profile of nivolumab monotherapy: A pooled analysis of patients with advanced melanoma," Journal of Clinical Oncology, vol. 35, no. 7, pp. 785-792, 2017.

[13] J. Schachter, A. Ribas, G. V. Long et al., "Pembrolizumab versus ipilimumab for advanced melanoma: final overall survival results of a multicentre, randomised, open-label phase 3 study (KEYNOTE-006)," The Lancet, vol. 390, no. 10105, pp. 1853$1862,2017$.

[14] F. S. Hodi, V. Chiarion-Sileni, R. Gonzalez et al., "Nivolumab plus ipilimumab or nivolumab alone versus ipilimumab alone in advanced melanoma (CheckMate 067): 4-year outcomes of a multicentre, randomised, phase 3 trial," The Lancet Oncology, vol. 19, no. 11, pp. 1480-1492, 2018.

[15] YJ. Jansen, EA. Rozeman, and R. Mason, "SMR Congress 2017 abstracts," Pigment Cell \& Melanoma Research, vol. 31, no. 1, pp. 125-230, 2018.

[16] S. A. Christiansen, D. Swoboda, K. Gardner, S. Rapisuwon, M. B. Atkins, and G. T. Gibney, "Off treatment survival (OTS) in patients (pts) with advanced melanoma after anti-PD1 therapy," Journal of Clinical Oncology, vol. 36, no. supplement 15, p. 9554, 2018.

[17] K. Nguyen, R. Mason, R. Ladwa et al., "Relapse after cessation of PD-1 based therapy for complete responders in metastatic melanoma," Journal of Clinical Oncology, vol. 36, no. 15_suppl, pp. 9536-9536, 2018.

[18] A. C. Tan, L. Emmett, S. Lo et al., "Utility of 1-year FDGPET (PET) to determine outcomes from anti-PD-1 (PD1) based therapy in patients (pts) with metastatic melanoma (MM)," Journal of Clinical Oncology, vol. 36, no. 15_suppl, p. 9517, 2018.

[19] G. Schvartsman, J. Ma, R. L. Bassett et al., "Outcomes of metastatic melanoma (MM) patients (pts) after discontinuation 
of anti-Programmed-Death 1 (PD1) therapy without disease progression," Journal of Clinical Oncology, vol. 36, supplement 15, p. 9549, 2018.

[20] M. A. Postow and M. D. Hellmann, "Adverse events associated with immune checkpoint blockade," The New England Journal of Medicine, vol. 378, no. 12, pp. 1163-1165, 2018.

[21] M. A. Postow, R. Sidlow, and M. D. Hellmann, "Immune-related adverse events associated with immune checkpoint blockade," The New England Journal of Medicine, vol. 378, no. 2, pp. 158$168,2018$.

[22] J. S. Weber, M. Postow, C. D. Lao, and D. Schadendorf, "Management of adverse events following treatment with antiprogrammed death-1 agents," The Oncologist, vol. 21, no. 10, pp. 1230-1240, 2016.

[23] D. B. Johnson, D. L. Friedman, E. Berry et al., "Survivorship in immune therapy: Assessing chronic immune toxicities, health outcomes, and functional status among long-term ipilimumab survivors at a single referral center," Cancer Immunology Research, vol. 3, no. 5, pp. 464-469, 2015.

[24] A. T. Faje, R. Sullivan, D. Lawrence et al., "Ipilimumab-induced hypophysitis: a detailed longitudinal analysis in a large cohort of patients with metastatic melanoma," The Journal of Clinical Endocrinology \& Metabolism, vol. 99, no. 11, pp. 4078-4085, 2014.

[25] F. Albarel, C. Gaudy, F. Castinetti et al., "Long-term follow-up of ipilimumab-induced hypophysitis, a common adverse event of the anti-CTLA-4 antibody in melanoma," European Journal of Endocrinology, vol. 172, no. 2, pp. 195-204, 2015.

[26] C. Lebbé, N. Meyer, L. Mortier et al., "Evaluation of two dosing regimens for nivolumab in combination with ipilimumab in patients with advanced melanoma: results from the phase IIIb/IV checkmate 511 trial," Journal of Clinical Oncology, 2019.

[27] G. V. Long, V. Atkinson, J. S. Cebon et al., "Standard-dose pembrolizumab in combination with reduced-dose ipilimumab for patients with advanced melanoma (KEYNOTE-029): an open-label, phase $1 \mathrm{~b}$ trial," The Lancet Oncology, vol. 18, no. 9, pp. 1202-1210, 2017.

[28] N. K. Aaronson, S. Ahmedzai, B. Bergman et al., "The European Organization for Research and Treatment of Cancer QLQ-C30: a quality-of-life instrument for use in international clinical trials in oncology," Journal of the National Cancer Institute, vol. 85, no. 5, pp. 365-376, 1993.

[29] B. J. Zebrack, P. A. Ganz, C. A. Bernaards, L. Petersen, and L. Abraham, "Assessing the impact of cancer: Development of a new instrument for long-term survivors," Psycho-Oncology, vol. 15, no. 5, pp. 407-421, 2006.

[30] D. F. Cella, D. S. Tulsky, G. Gray et al., "The functional assessment of cancer therapy scale: development and validation of the general measure," Journal of Clinical Oncology, vol. 11, no. 3, pp. 570-579, 1993.

[31] J. N. Cormier, M. I. Ross, J. E. Gershenwald et al., "Prospective assessment of the reliability, validity, and sensitivity to change of the functional assessment of cancer therapy-melanoma questionnaire," Cancer, vol. 112, no. 10, pp. 2249-2257, 2008.

[32] M. van Leeuwen, O. Husson, P. Alberti et al., "Understanding the quality of life (QOL) issues in survivors of cancer: towards the development of an EORTC QOL cancer survivorship questionnaire," Health and Quality of Life Outcomes, vol. 16, no. 1, p. 114, 2018.

[33] D. A. Revicki, A. J. M. van den Eertwegh, P. Lorigan et al., "Health related quality of life outcomes for unresectable stage
III or IV melanoma patients receiving ipilimumab treatment," Health and Quality of Life Outcomes, vol. 10, p. 66, 2012.

[34] D. Schadendorf, R. Dummer, A. Hauschild et al., "Healthrelated quality of life in the randomised KEYNOTE-002 study of pembrolizumab versus chemotherapy in patients with ipilimumab-refractory melanoma," European Journal of Cancer, vol. 67, pp. 46-54, 2016.

[35] D. Schadendorf, J. Larkin, J. Wolchok et al., "Health-related quality of life results from the phase III CheckMate 067 study," European Journal of Cancer, vol. 82, pp. 80-91, 2017.

[36] M. E. Beutel, S. Fischbeck, H. Binder et al., "Depression, anxiety and quality of life in long-term survivors of malignant melanoma: a register-based cohort study," PLoS ONE, vol. 10, no. 1, Article ID e0116440, 2015.

[37] O. Palesh, A. Aldridge-Gerry, K. Bugos et al., "Health behaviors and needs of melanoma survivors," Supportive Care in Cancer, vol. 22, no. 11, pp. 2973-2980, 2014.

[38] G. Schubert-Fritschle, A. Schlesinger-Raab, R. Hein et al., "Quality of life and comorbidity in localized malignant melanoma: results of a German population-based cohort study," International Journal of Dermatology, vol. 52, no. 6, pp. 693-704, 2013.

[39] Y. Hamama-Raz, Z. Solomon, J. Schachter, and E. Azizi, “Objective and subjective stressors and the psychological adjustment of melanoma survivors," Psycho-Oncology, vol. 16, no. 4, pp. 287294, 2007.

[40] Y. Hamama-Raz, "Does psychological adjustment of melanoma survivors differs between genders?” Psycho-Oncology, vol. 21, no. 3, pp. 255-263, 2012.

[41] A. Waldmann, S. Nolte, R. Pritzkuleit, E. W. Breitbart, and A. Katalinic, "Different aspects of self-reported quality of life in 450 German melanoma survivors," Cancers, vol. 3, no. 2, pp. 2316-2332, 2011.

[42] C. Holterhues, D. Cornish, L. V. Van De Poll-Franse et al., "Impact of melanoma on patients' lives among 562 survivors: a dutch population-based study," JAMA Dermatology, vol. 147, no. 2, pp. 177-185, 2011.

[43] S. Fischbeck, B. H. Imruck, M. Blettner et al., "Psychosocial care needs of melanoma survivors: Are they being met?" PLoS ONE, vol. 10, no. 8, Article ID e0132754, 2015.

[44] A. Schlesinger-Raab, G. Schubert-Fritschle, R. Hein et al., "Quality of life in localised malignant melanoma," Annals of Oncology, vol. 21, no. 12, pp. 2428-2435, 2010.

[45] K. D. Cromwell, Y. J. Chiang, J. Armer et al., "Is surviving enough? Coping and impact on activities of daily living among melanoma patients with lymphoedema," European Journal of Cancer Care, vol. 24, no. 5, pp. 724-733, 2015.

[46] J.-F. Hamel, M. Pe, C. Coens et al., "A systematic review examining factors influencing health related quality of life among melanoma cancer survivors," European Journal of Cancer, vol. 69, pp. 189-198, 2016.

[47] M. Dieng, N. A. Kasparian, A. E. Cust et al., "Sensitivity of preference-based quality-of-life measures for economic evaluations in early-stage Melanoma," JAMA Dermatology, vol. 154, no. 1, pp. 52-59, 2018.

[48] A. Bottomley, M. Pe, J. Sloan et al., "Analysing data from patientreported outcome and quality of life endpoints for cancer clinical trials: a start in setting international standards," The Lancet Oncology, vol. 17, no. 11, pp. e510-e514, 2016.

[49] G. T. Deimling, K. F. Bowman, S. Sterns, L. J. Wagner, and B. Kahana, "Cancer-related health worries and psychological 
distress among older adult, long-term cancer survivors," PsychoOncology, vol. 15, no. 4, pp. 306-320, 2006.

[50] T. M. Petrella, C. Robert, E. Richtig et al., "Patient-reported outcomes in KEYNOTE-006, a randomised study of pembrolizumab versus ipilimumab in patients with advanced melanoma," European Journal of Cancer, vol. 86, pp. 115-124, 2017.

[51] B. Löwe, K. Gräfe, S. Zipfel, S. Witte, B. Loerch, and W. Herzog, "Diagnosing ICD-10 depressive episodes: superior criterion validity of the Patient Health Questionnaire," Psychotherapy and Psychosomatics, vol. 73, no. 6, pp. 386-391, 2004.

[52] B. Löwe, O. Decker, S. Müller et al., "Validation and standardization of the generalized anxiety disorder screener (GAD-7) in the general population," Medical Care, vol. 46, no. 3, pp. 266274, 2008.

[53] T. A. Revenson, K. M. Schiaffino, S. Deborah Majerovitz, and A. Gibofsky, "Social support as a double-edged sword: The relation of positive and problematic support to depression among rheumatoid arthritis patients," Social Science \& Medicine, vol. 33, no. 7, pp. 807-813, 1991.

[54] J. M. Armer, M. H. Henggeler, C. W. Brooks, E. A. Zagar, S. Homan, and B. R. Stewart, "The health deviation of post-breast cancer lymphedema: symptom assessment and impact on selfcare agency," Self-Care, Dependent-Care \& Nursing, vol. 16, no. 1, pp. 14-21, 2008.

[55] P. Hopwood, I. Fletcher, A. Lee, and S. Al Ghazal, "A body image scale for use with cancer patients," European Journal of Cancer, vol. 37, no. 2, pp. 189-197, 2001.

[56] S. Greer and M. Watson, "Mental adjustment to cancer: its measurement and prognostic importance," Cancer Surveys, vol. 6, no. 3, pp. 439-453, 1987.

[57] C. T. Veit and J. E. Ware, "The structure of psychological distress and well-being in general populations," Journal of Consulting and Clinical Psychology, vol. 51, no. 5, pp. 730-742, 1983.

[58] T. A. Kessler, "The Cognitive Appraisal of Health Scale: development of psychometric evaluation," Research in Nursing \& Health, vol. 21, no. 1, pp. 73-82, 1998.

[59] J. E. Ware and C. D. Sherbourne, "The MOS 36-item shortform health survey (SF-36): I. Conceptual framework and item selection," Medical Care, vol. 30, no. 6, pp. 473-483, 1992.

[60] C. M. Crespi, P. A. Ganz, L. Petersen, A. Castillo, and B. Caan, "Refinement and psychometric evaluation of the impact of cancer scale," Journal of the National Cancer Institute, vol. 100, no. 21, pp. 1530-1541, 2008.

[61] J. Richardson, A. Iezzi, M. A. Khan, and A. Maxwell, "Validity and reliability of the assessment of quality of life (AQoL)-8D multi-attribute utility instrument," The Patient, vol. 7, no. 1, pp. 85-96, 2014.

[62] S. Simard and J. Savard, "Fear of Cancer Recurrence Inventory: Development and initial validation of a multidimensional measure of fear of cancer recurrence," Supportive Care in Cancer, vol. 17, no. 3, pp. 241-251, 2009.

[63] C. Loquai, V. Scheurich, N. Syring et al., "Screening for distress in routine oncological care-a survey in 520 melanoma patients," PLoS ONE, vol. 8, no. 7, Article ID e66800, 2013.

[64] A. J. Roth, A. B. Kornblith, L. Batel-Copel, E. Peabody, H. I. Scher, and J. C. Holland, "Rapid screening for psychologic distress in men with prostate carcinoma: A pilot study," Cancer, vol. 82, no. 10, pp. 1904-1908, 1998.

[65] J. D. Wolchok, B. Neyns, G. Linette et al., "Ipilimumab monotherapy in patients with pretreated advanced melanoma: a randomised, double-blind, multicentre, phase 2, dose-ranging study," The Lancet Oncology, vol. 11, no. 2, pp. 155-164, 2010.

[66] C. Robert, D. Schadendorf, M. Messina, F. S. Hodi, and S. O'Day, "Efficacy and safety of retreatment with ipilimumab in patients with pretreated advanced melanoma who progressed after initially achieving disease control," Clinical Cancer Research, vol. 19, no. 8, pp. 2232-2239, 2013.

[67] J. D. Wolchok, A. Hoos, S. O’Day et al., “Guidelines for the evaluation of immune therapy activity in solid tumors: immune-related response criteria," Clinical Cancer Research, vol. 15, no. 23, pp. 7412-7420, 2009.

[68] J. S. Weber, K. C. Kähler, and A. Hauschild, "Management of immune-related adverse events and kinetics of response with ipilimumab," Journal of Clinical Oncology, vol. 30, no. 21, pp. 2691-2697, 2012.

[69] P. Ascierto, L. Bastholt, P. Mohr et al., "EURO-VOYAGE: Effectiveness and safety of ipilimumab (IPI) administered during a European Expanded Access Programme (EAP) in patients with advanced melanoma (MEL)," European Journal of Cancer, vol. 72, p. S128, 2017.

[70] A. Martens, K. Wistuba-Hamprecht, M. G. Foppen et al., "Baseline peripheral blood biomarkers associated with clinical outcome of advanced melanoma patients treated with ipilimumab," Clinical Cancer Research, vol. 22, no. 12, pp. 2908-2918, 2016.

[71] S. Kelderman, B. Heemskerk, H. van Tinteren et al., "Lactate dehydrogenase as a selection criterion for ipilimumab treatment in metastatic melanoma," Cancer Immunology, Immunotherapy, vol. 63, no. 5, pp. 449-458, 2014.

[72] J. D. Wolchok, V. Chiarion-Sileni, R. Gonzalez et al., "Overall survival with combined nivolumab and ipilimumab in advanced melanoma," The New England Journal of Medicine, vol. 377, no. 14, pp. 1345-1356, 2017.

[73] C. Robert, G. V. Long, B. Brady et al., "Nivolumab in previously untreated melanoma without BRAF mutation," The New England Journal of Medicine, vol. 372, no. 4, pp. 320-330, 2015.

[74] J. Larkin, V. Chiarion-Sileni, R. Gonzalez et al., "Combined nivolumab and ipilimumab or monotherapy in untreated melanoma," The New England Journal of Medicine, vol. 373, no. 1, pp. 23-34, 2015.

[75] C. Robert, J. Schachter, G. V. Long et al., "Pembrolizumab versus ipilimumab in advanced melanoma," The New England Journal of Medicine, vol. 372, no. 26, pp. 2521-2532, 2015.

[76] C. Robert, A. Ribas, O. Hamid et al., "Durable complete response after discontinuation of pembrolizumab in patients with metastatic melanoma," Journal of Clinical Oncology, vol. 36, no. 17, pp. 1668-1674, 2018.

[77] S. Paltrinieri, S. Fugazzaro, L. Bertozzi et al., "Return to work in European Cancer survivors: a systematic review," Supportive Care in Cancer, vol. 26, no. 9, pp. 2983-2994, 2018.

[78] A. C. Kirchhoff, K. R. Krull, K. K. Ness et al., "Physical, mental, and neurocognitive status and employment outcomes in the childhood cancer survivor study cohort," Cancer Epidemiology Biomarkers \& Prevention, vol. 20, no. 9, pp. 1838-1849, 2011.

[79] J. McLoone, K. Watts, S. Menzies, B. Meiser, P. Butow, and N. Kasparian, "When the risks are high: Psychological adjustment among melanoma survivors at high risk of developing new primary disease," Qualitative Health Research, vol. 22, no. 8, pp. 1102-1113, 2012.

[80] N. A. Kasparian, J. K. McLoone, and P. N. Butow, "Psychological responses and coping strategies among patients with malignant 
melanoma: a systematic review of the literature," JAMA Dermatology, vol. 145, no. 12, pp. 1415-1427, 2009.

[81] M. Dieng, P. N. Butow, D. S. J. Costa et al., "Psychoeducational intervention to reduce fear of cancer recurrence in people at high risk of developing another primarymelanoma: results of a randomized controlled trial," Journal of Clinical Oncology, vol. 34, no. 36, pp. 4405-4414, 2016.

[82] S. Du Four, Y. Janssen, A. Michotte et al., "Focal radiation necrosis of the brain in patients with melanoma brain metastases treated with pembrolizumab," Cancer Medicine, vol. 7, no. 10, pp. 4870-4879, 2018.

[83] A. Rogiers, G. Awada, J. K. Schwarze et al., "Emotional and cognitive disturbances in long-term melanoma survivors treated with ipilimumab," Journal of Clinical Oncology, vol. 37, Supplement 8, Abstract 97, 2019.

[84] A. Rogiers, J. De Cremer, L. Ben Salama et al., "Psychosocial outcome and health-related quality of life (HRQoL) in advanced melanoma survivors," Journal of Clinical Oncology, vol. 36, supplement 7, p. 162, 2018. 


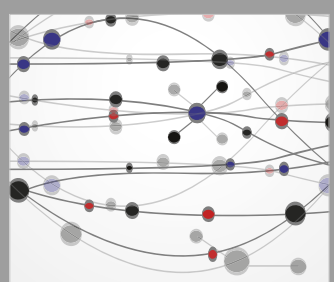

The Scientific World Journal
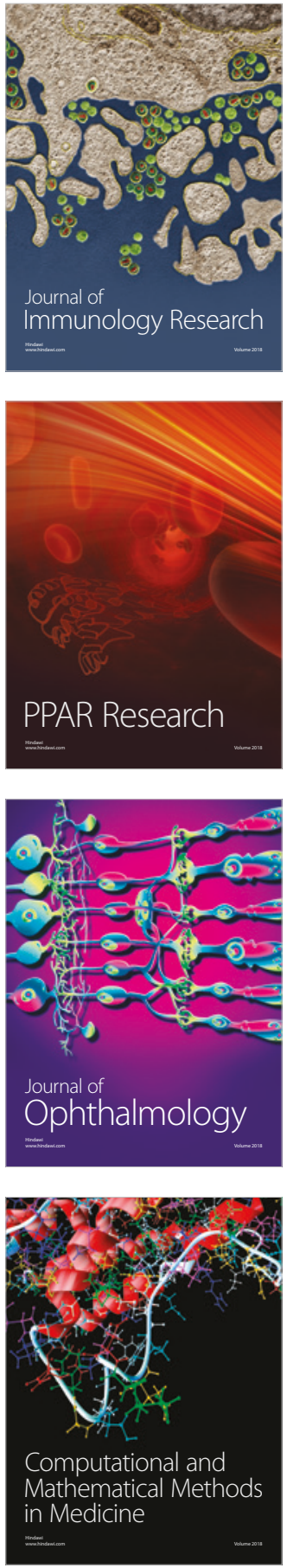

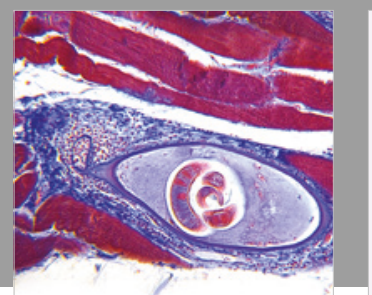

Gastroenterology Research and Practice

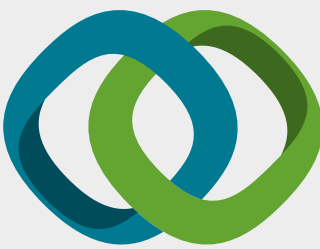

\section{Hindawi}

Submit your manuscripts at

www.hindawi.com
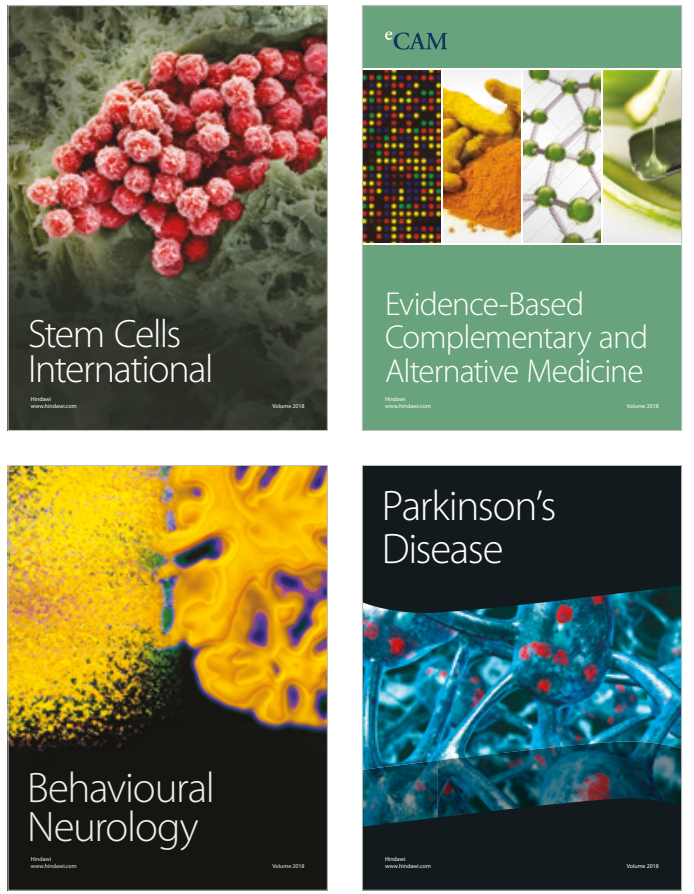

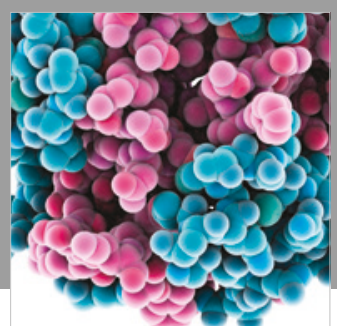

ournal of

Diabetes Research

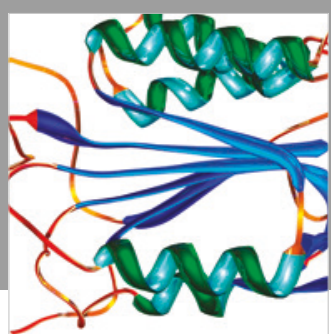

Disease Markers
